\title{
Estimating Body Response of Acoustic Guitar Notes using Adaptive Cepstral Domain Windowing
}

\author{
Minakshi Pradeep Atre ${ }^{{ }^{*}}$ and Shaila D. Apte ${ }^{2}$ \\ 'Department of Electronics and Telecommunication PVG's COET, JSPM's RSCOE, Pune - 411033, \\ Maharastra, India; mpatre29@gmaill.com \\ 2Department of Electronics and Telecommunication, JSPM's RSCOE, Pune - 411033, \\ Maharastra, India; sdapte@rediffmail.com
}

\begin{abstract}
Understanding an acoustic guitar by analyzing music notes has been the focus of proposed work. It started with calculation and verification of octave relationship of frequencies along frets of a string. The mathematical analysis of frequency triggered the curiosity to analyze music notes in cepstral domain. Cepstral domain was helpful to estimate the impulse response ie body response of music note played on that fret. Truncating the samples for body response, gave excitation signals. Ultimately, convolution of this estimated impulse response and the separated excitation signals resulted into synthesized notes. To get the highly correlated synthesized notes, the number of samples in a window was varied. The number of samples giving maximum similarity will be the optimum number of samples for that music note. They vary from fret to fret. So the name is Adaptive Cepstral Domain Windowing Method (ACDW). The size of the window is changed. The algorithm is run for 25 different values of samples, ranging from 50 to 300 . Finally, the best one is chosen for synthesis of music notes. Objectives: The focus of the work is estimate the guitar body response by using cepstral domain. Methods/Statistical Analysis: The guitar notes for all twenty frets of all six strings have been recorded with $16 \mathrm{kHz}$ as the sampling rate. This resulted into database of 120 notes for each playing style. The researchers have proposed different methods to find the impulse response of guitar notes. One method includes recording the impulse response by pulling the string till it breaks. Another one is hammer method. The work proposed here uses signal processing technique such as cepstral domain to find the impulse response. The synthesis is been carried out to check if the estimated impulse response can be used to synthesize the music note. Findings: Many scientists have modeled acoustic guitar notes. There are various methods for finding the impulse response as well. Though the cepstral domain approach is commonly used in speech synthesis, this is used for music notes in unique way to find the body response without breaking the guitar strings. Application/Improvements: The method is used for finding the body response of acoustic guitar. The work is focused to new approach towards music note synthesis.
\end{abstract}

Keywords: Acoustic Guitar, Adaptive Window, Body Response, Cepstral Domain

\section{Introduction}

The string instruments are mainly classified as plucked string instruments and bowed string instruments. The work is focused on plucked stringed instrument ie box shaped acoustic guitar. Guitar has two main parts: a set of strings and a soundboard. Plucking the string gives excitation to it. This excitation signal gets convolved with impulse response of the guitar body. Soundboard of guitar body acts as a resonator and amplifies the sound note generated by plucking the string either by finger or pick.

Figure 1 depicts the acoustic guitar body structure. An acoustic guitar consists of six strings. Each string has 18 to 21 frets. The symbol ' $x$ ' represents the fret number. These strings are tied between nut and the bridge. The guitar body has a round hole in the middle of guitar body

*Author for correspondence 
acting as resonance cavity. Music samples for each fret and each string for FAW 802 guitar model are recorded at $16 \mathrm{kHz}$ sampling frequency. This guitar consists of 21 frets. $21^{\text {st }}$ fret music note is not used for analysis. The artist was recorded for picked (using plectrum) and plucked (using finger) sound notes. Being an artist of Guitar, it motivated the author to study the Guitar notes in different domains.

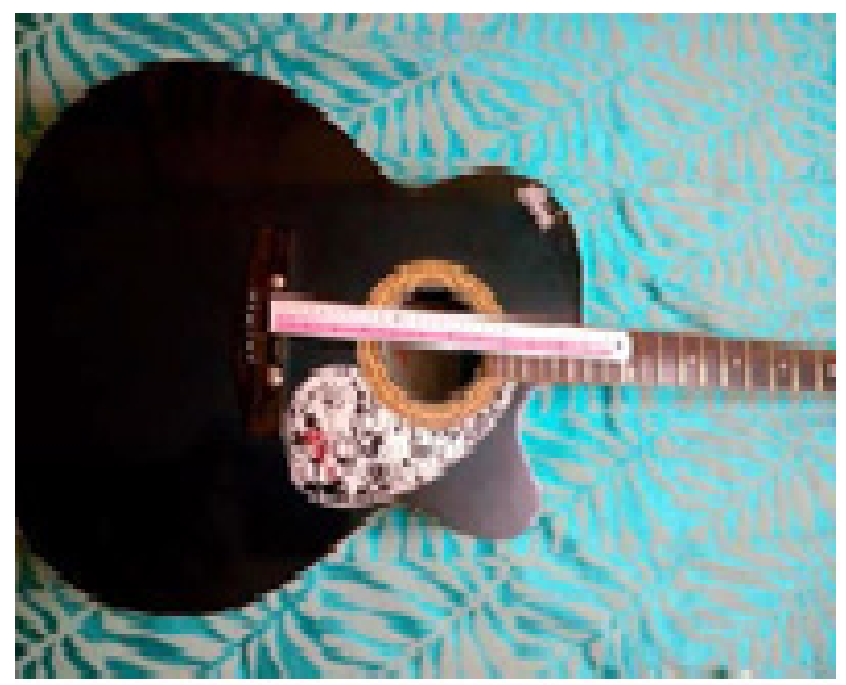

Figure 1. Acoustic Guitar Body Structure.

The paper is organized as follows. Next section gives overview of work done by different researchers. Section II covers the detailed discussion of Adaptive Cepstral Domain Windowing (ACDW) method. Section III concludes the work.

Many researchers have analyzed and synthesized guitar notes. Some of them have developed different methods to model the string instruments to achieve greater improvements in the quality of virtual string instruments. Impulse response method is one of them. In reference ${ }^{1}$, the researchers analyzed stringed instruments by testing their string motion. The factors like string tension, mass per unit length and string length influence fundamental tone of vibration of guitar. The authors performed vibration analysis for the steel and nylon string types. Major focus was analysis of effects of string type on the frequency spectra along with the same plucking position for both string types. This motivated to collect the music notes for different plucking styles and observe their body response nature. In reference ${ }^{2}$ they used the joint source filter approach for modeling the guitar tones. Here the filter order poses limitations for modeling tones of guitar. While K. Lanc analyzed the string motion, the scientists $\underline{3}^{3}$ proposed the efficient computational model for stringed instruments based on body response. The work focused on body modeling to estimate the least damped and most damped modes of resonance. The body model was factored into three main blocks as excitation, resonator and string. They implemented commuted synthesis for stringed instruments. The work has been extended by the same authors to propose the digital waveguide model for string instrument as discussed ${ }^{4-5}$. On the similar lines ${ }^{6}$ introduced novel impulse response measurement method for stringed instruments. The method involved exciting the dampened strings at the bowing in bowed instruments and plucking strings in plucked instruments by a copper wire. This copper wire was pulled to such an extent at the time of excitation that it would make it break. Then considering the string deflection, impulse responses were measured. Here the focus was on to bowed instruments for impulse response measurement. $\operatorname{In}^{7}$ the authors discussed feature based extraction of different plucking and expression styles for electric bass guitar. Their focus was estimation of excitation function rather body response. The important point they proposed was estimation of best suitable excitation function to be used to model the input signal. Although this was for electric bass guitar, the importance of suitable excitation function held true for modeling the whole family of stringed instruments. The authors in $\frac{8-10}{}$ put another angle to guitar modeling by considering the player-instrument interaction parameters: touch and collisions. They used the digital waveguide technique for synthesis with addition of insertion of simple scattering junctions at various points along the digital waveguide. The work proposed in $\underline{11}$ by the authors has focus on the finer level of identifying the playing defects. This rather considers the player interaction based analysis of music notes.

The area of focus for all music researchers being instrument modeling, the work proposed here also focused on modeling of acoustic guitar with reference to cepstral domain. It motivated the author to analyze the acoustic guitar notes in frequency domain and then quefrency domain. It proposed an impulse response estimation method for acoustic guitar and is based on calculation of excitation function and guitar body response for box shaped acoustic guitar. As described in ${ }^{6}$, to estimate the impulse response, the string is pulled to such as extent that it gets broken. Cepstral method to estimate the impulse response was without the need to break the string. Cepstral domain analysis is used in speech analysis 
but to best of our knowledge, the cepstral domain impulse response modeling for musical notes is not been used for synthesis of guitar notes. Although cepstral domain is popular approach, it gives innovative way to calculate body response of musical instruments.

\section{Methodology}

An impulse response can be considered as the fingerprint of the musical instrument. There are different approaches for calculation of impulse response as discussed in introduction section. Impulse hammer method is the standard one. Another method involves stretching the string of a copper wire till it breaks as mentioned earlier in ${ }^{6}$. Stretching the copper wire till it breaks poses limitations. The work discusses the approach to find an impulse response or body response by different approach without breaking the string or the hammer method.

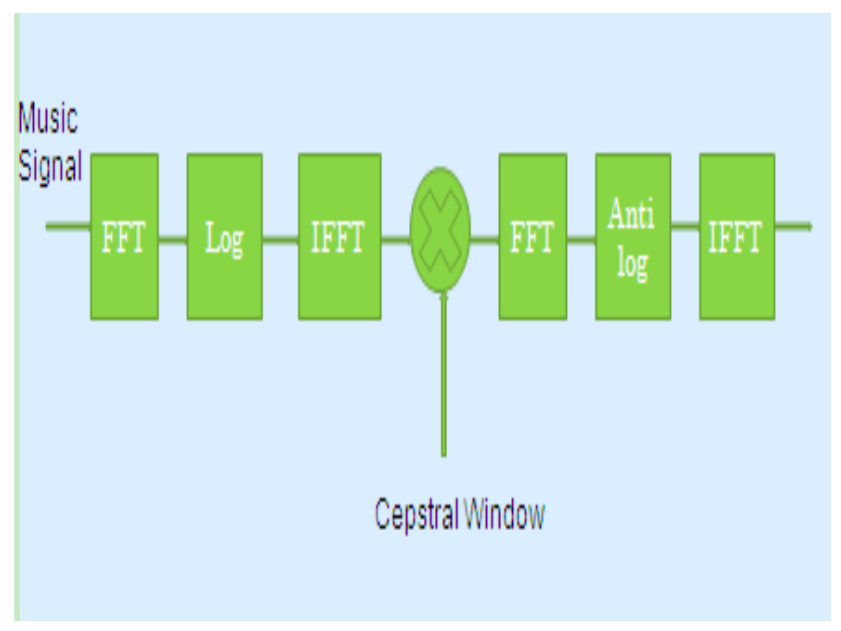

Figure 2. Block Schematic for impulse response using Cepstral Windowing.

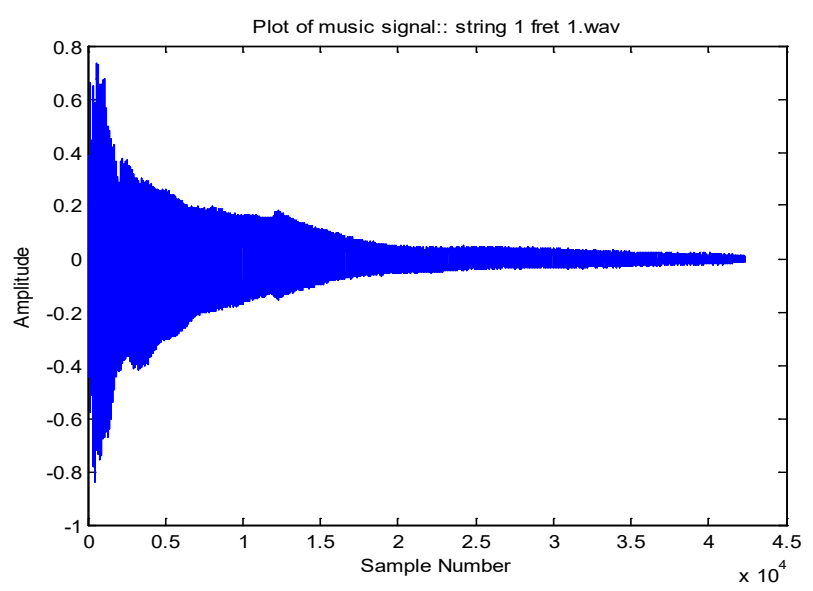

Figure 3. Plot of the music note: string $\mathrm{E}$ fret 1 finger plucked note.

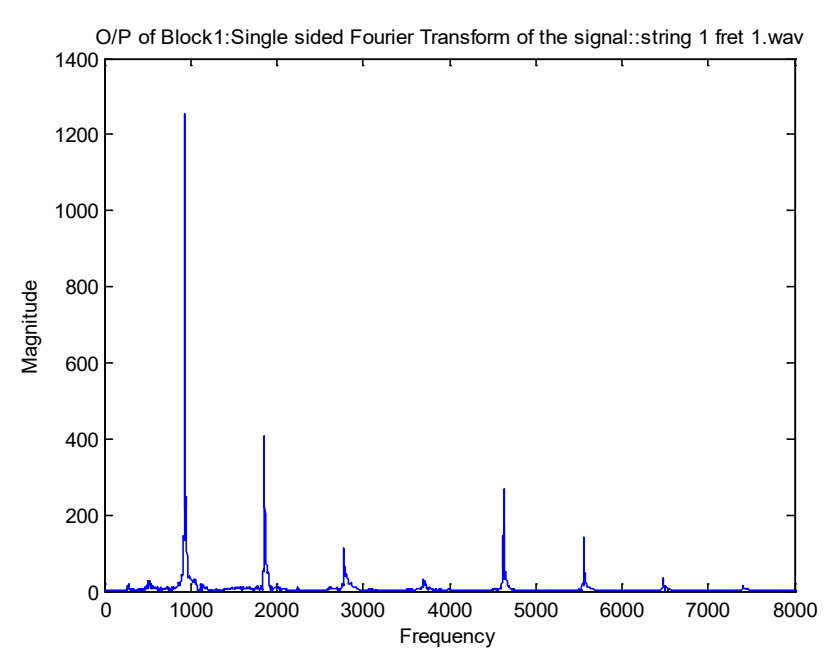

Figure 4. FFT of string E fret 1 finger plucked note.

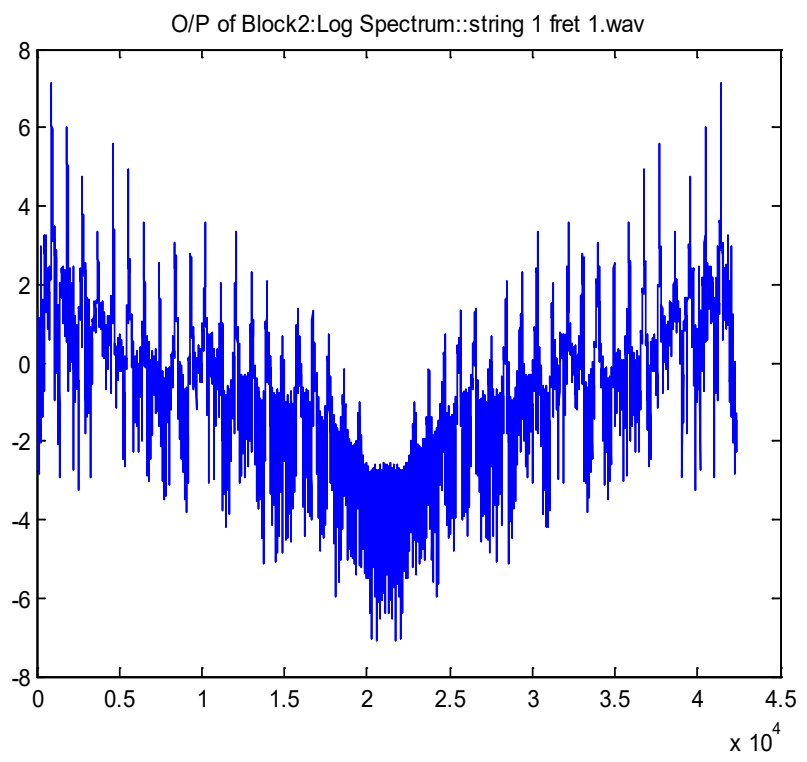

Figure 5. Log of FFT of string E fret 1 finger plucked music note.

It is the novel approach towards calculation of body response using cepstral domain. The Figure 2 shows the block schematic for calculation of impulse response using cepstral domain. The music notes are the input to this block schematic. As mentioned in introduction, the music notes have been recorded for two plucking styles and the analysis is carried out for both plucking styles. Referring to Figure 2 block schematic, first the guitar note is fed as an input. Figure 3 gives the time domain plot of input music note, string E fret 1 finger plucked. Then FFT block calculates the spectrum of the guitar signal. Figure 4 shows the Fourier Transform of string E fret 1 finger plucked guitar note. Then complex 
logarithm of FFT spectrum is calculated. This is depicted in Figure 5. Now IFFT is calculated and the signal enters in cepstral domain in which the periodic excitation is a rapidly varying function and guitar body response appears as the envelope of the spectrum that is a slowly varying function.

All the plots are named with string number and then fret number e.g. string $\mathrm{E}$ and then fret 1 . The extension for the note is 'pick' for plectrum plucked notes and extension 'finger' is for finger plucked notes. The cepstral domain analysis is mainly focused to: Impulse response calculation of acoustic guitar body for different notes for plucked style and Excitation signal calculation for all frets and strings.

\section{Results and Discussion}

The aim is to find the impulse response of guitar body. So, the window is applied to the cepstral domain signal to separate the impulse response of guitar from the excitation signal. The cepstrum contains a cluster near the origin corresponding to the impulse response of the body and the high time region consists of the excitation signal. This can be clearly seen in Figure 6. The plot shows only 2000 samples. If the complete cepstrum is plotted, it will show the symmetry of waveform indicating same periodic nature in the end. A low time window is applied and all notes are analyzed for reconstruction. The samples in window are varied from 50 to 300 . This is with reference to Table 1 . The purpose of variation in number of samples is to separate the guitar body response and excitation signal as accurately as possible. And this accuracy is been decided by observing the correlation coefficient values. Correlation values give the similarity coefficient between the original music note and the synthesized note. Figure 7 shows the body response plot for string $\mathrm{E}$ fret 1 music note after separation. In summary, all frets of string $\mathrm{E}$ are analyzed, plucked and picked music notes by taking those varying number of samples of the cluster near origin.

Now first 50 samples (specifically applicable to string E fret 1 finger plucked note) for string $E$ fret 1 finger plucked note are retained and then again FFT is taken, as shown in Figure 8. An antilog is taken, Figure 9 and finally the inverse FFT is plotted, shown in Figure 10. This resulted into the separation of guitar body response from the rapidly varying envelope of the excitation signal.
Secondly, same set of samples i.e 50 samples is made zero and then set of remaining samples is analyzed by taking FFT, then antilog is calculated and finally the inverse FFT, depicted in Figure 11. This cluster of samples resulted into separation of excitation signal from the guitar body response.

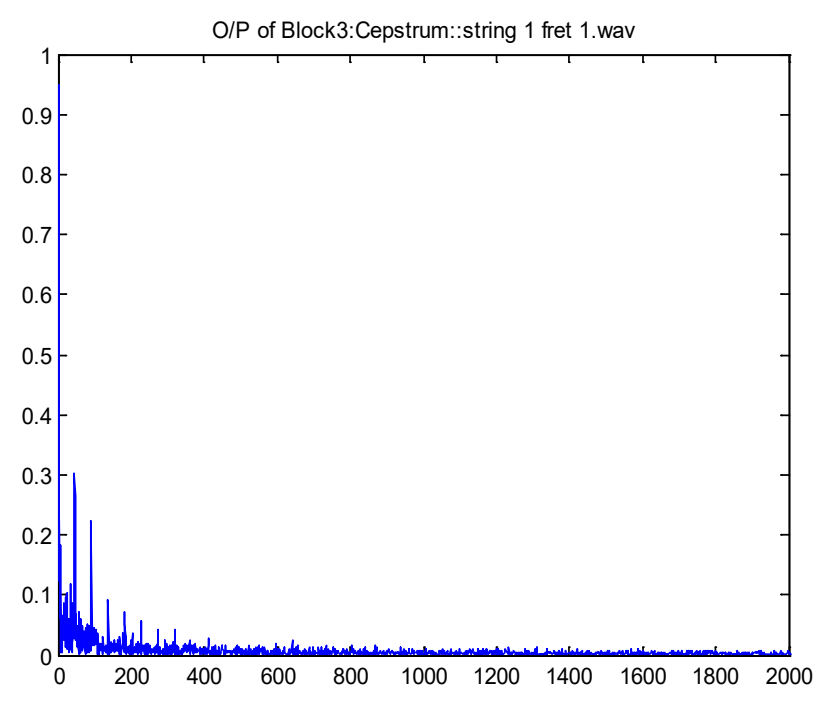

Figure 6. IFFT of $\log$ of string $\mathrm{E}$ fret 1 finger plucked music note.

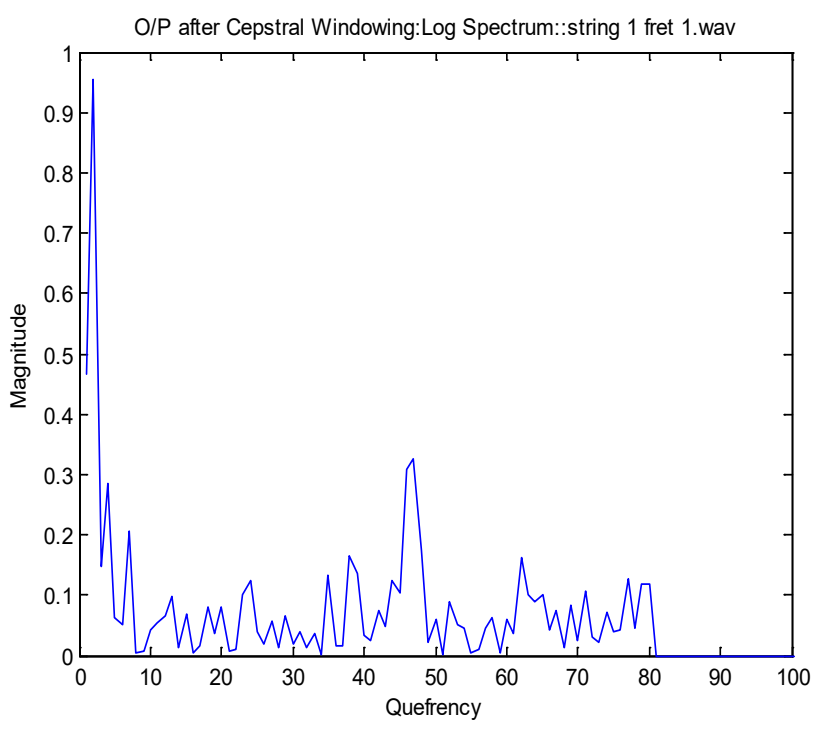

Figure 7. Output after applying cepstral window of 50 samples. 
Table 1. Correlation coefficient values for frets of string E with finger plucked notes

\begin{tabular}{|c|c|c|c|c|c|c|c|}
\hline String E & Open string & Fret1 & Fret 2 & Fret3 & Fret4 & Fret5 & Fret6 \\
\hline & 70 & 60 & 60 & 140 & 140 & 50 & 50 \\
\hline & \multicolumn{7}{|c|}{ Optimum Number of samples } \\
\hline & 0.9512 & 0.9596 & 0.9517 & 0.9361 & 0.8079 & 0.8856 & 0.9008 \\
\hline $\begin{array}{c}\text { Number of } \\
\text { Samples }\end{array}$ & \multicolumn{7}{|c|}{ Final Correlation Coefficient } \\
\hline 50 & 0.8943 & 0.9125 & 0.9171 & 0.7252 & 0.7676 & 0.8856 & 0.9008 \\
\hline 60 & 0.9471 & 0.9596 & 0.9517 & 0.7205 & 0.7188 & 0.8681 & 0.8622 \\
\hline 70 & 0.9512 & 0.9328 & 0.9394 & 0.7881 & 0.6401 & 0.8683 & 0.8285 \\
\hline 80 & 0.9507 & 0.9399 & 0.9283 & 0.8193 & 0.6712 & 0.8039 & 0.8703 \\
\hline 90 & 0.9329 & 0.9191 & 0.9406 & 0.8635 & 0.6217 & 0.8352 & 0.8741 \\
\hline 100 & 0.9086 & 0.9283 & 0.9441 & 0.8501 & 0.5738 & 0.8174 & 0.868 \\
\hline 110 & 0.926 & 0.9037 & 0.9274 & 0.847 & 0.5592 & 0.7963 & 0.8596 \\
\hline 120 & 0.9182 & 0.9098 & 0.9236 & 0.8045 & 0.5393 & 0.8106 & 0.8681 \\
\hline 130 & 0.9418 & 0.9309 & 0.8829 & 0.8833 & 0.5938 & 0.8116 & 0.8591 \\
\hline 140 & 0.9362 & 0.9144 & 0.8902 & 0.9361 & 0.8079 & 0.8106 & 0.8368 \\
\hline 150 & 0.9348 & 0.9193 & 0.8962 & 0.9324 & 0.7876 & 0.8004 & 0.8415 \\
\hline 160 & 0.9275 & 0.9213 & 0.886 & 0.9334 & 0.7615 & 0.8085 & 0.8414 \\
\hline 170 & 0.9272 & 0.9256 & 0.9071 & 0.9283 & 0.7364 & 0.8143 & 0.8288 \\
\hline 180 & 0.9275 & 0.9092 & 0.8719 & 0.9218 & 0.7253 & 0.799 & 0.8317 \\
\hline 190 & 0.9221 & 0.898 & 0.8699 & 0.919 & 0.7747 & 0.7967 & 0.837 \\
\hline 200 & 0.9244 & 0.8765 & 0.8479 & 0.9218 & 0.7897 & 0.8075 & 0.8372 \\
\hline 210 & 0.9153 & 0.8698 & 0.8376 & 0.9241 & 0.7919 & 0.7904 & 0.8416 \\
\hline 220 & 0.9124 & 0.8808 & 0.838 & 0.9266 & 0.797 & 0.7898 & 0.8347 \\
\hline 230 & 0.904 & 0.8674 & 0.8362 & 0.9247 & 0.7911 & 0.8018 & 0.8229 \\
\hline 240 & 0.9025 & 0.8539 & 0.8525 & 0.9312 & 0.7823 & 0.8033 & 0.8322 \\
\hline 250 & 0.9033 & 0.8597 & 0.8488 & 0.9205 & 0.777 & 0.8066 & 0.8384 \\
\hline 260 & 0.9075 & 0.8503 & 0.8259 & 0.9212 & 0.7765 & 0.8029 & 0.8319 \\
\hline 270 & 0.9002 & 0.8547 & 0.8238 & 0.9236 & 0.7739 & 0.8051 & 0.8279 \\
\hline 280 & 0.8974 & 0.8531 & 0.8355 & 0.9212 & 0.7744 & 0.8043 & 0.8302 \\
\hline 290 & 0.8765 & 0.8566 & 0.8335 & 0.914 & 0.7515 & 0.8025 & 0.8279 \\
\hline 300 & 0.8781 & 0.8564 & 0.8229 & 0.9093 & 0.7495 & 0.8061 & 0.834 \\
\hline
\end{tabular}




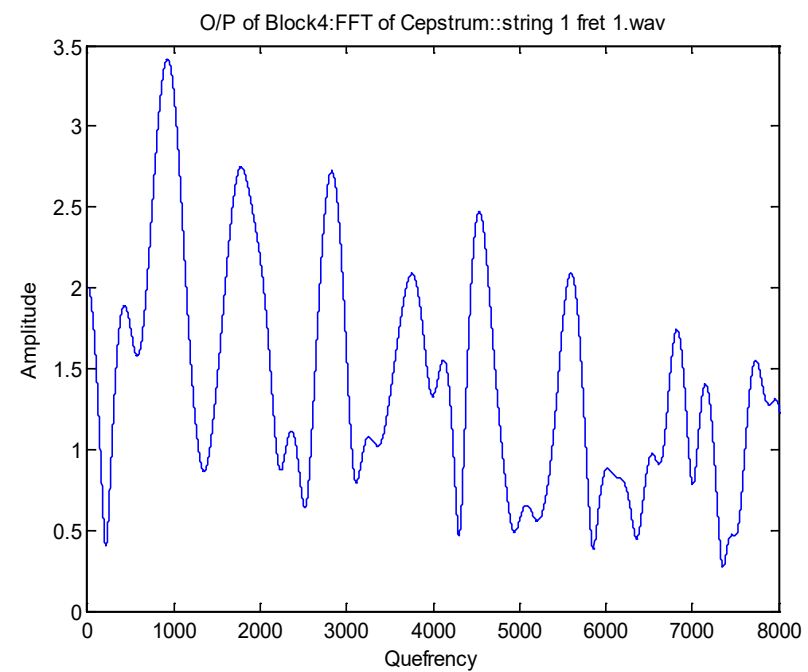

Figure 8. FFT of cepstrum after applying window of 50 samples.

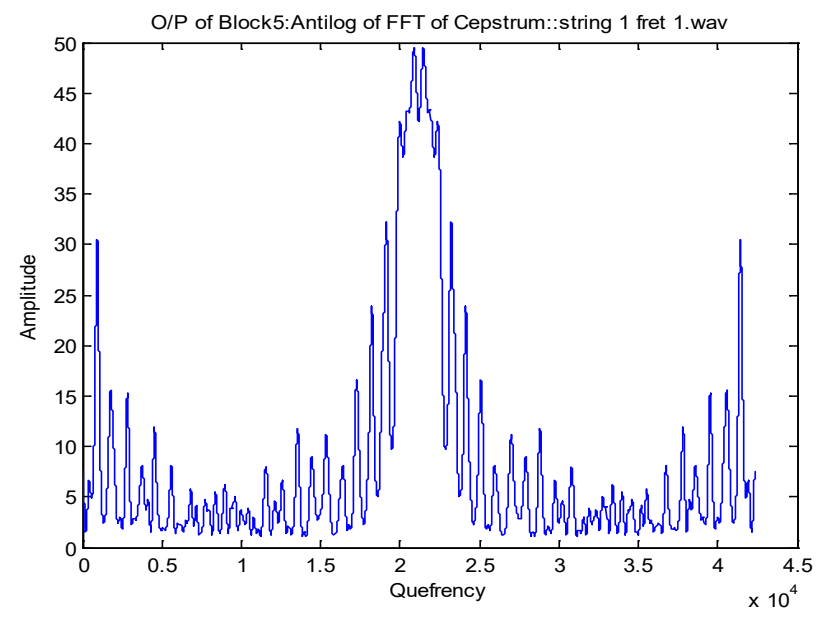

Figure 9. Antilog of windowed cepstrum for string E fret 1 finger note.

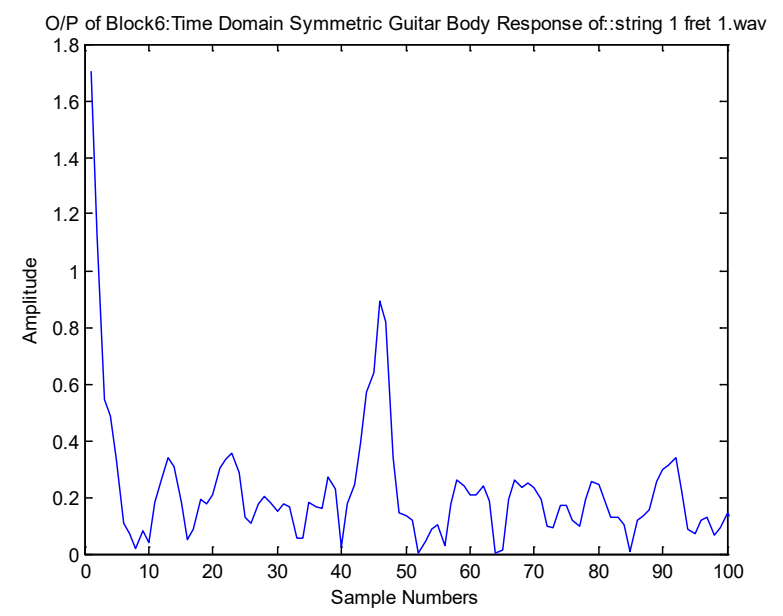

Figure 10. Final output of cepstral domain method, body response of guitar.

6 | Vol 11 (31)| August 2018| www.indjst.org

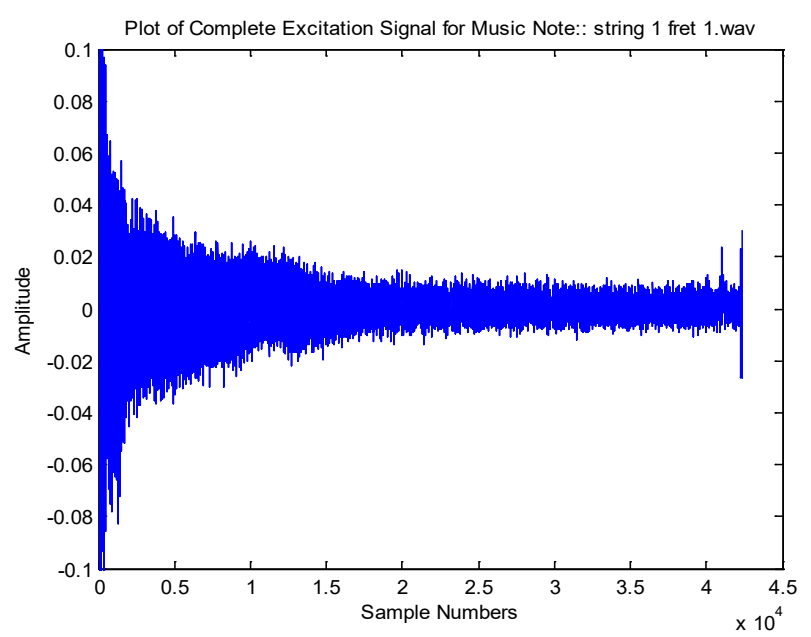

Figure 11. Time domain plot of excitation signal.

\subsection{Adaptive Windowing Approach}

To summarize the method proposed here: the music signal is taken as an input, FFT is calculated, logarithm is taken, IFFT is calculated, a window of suitable number of samples is applied, again an FFT, antilog and IFFT is

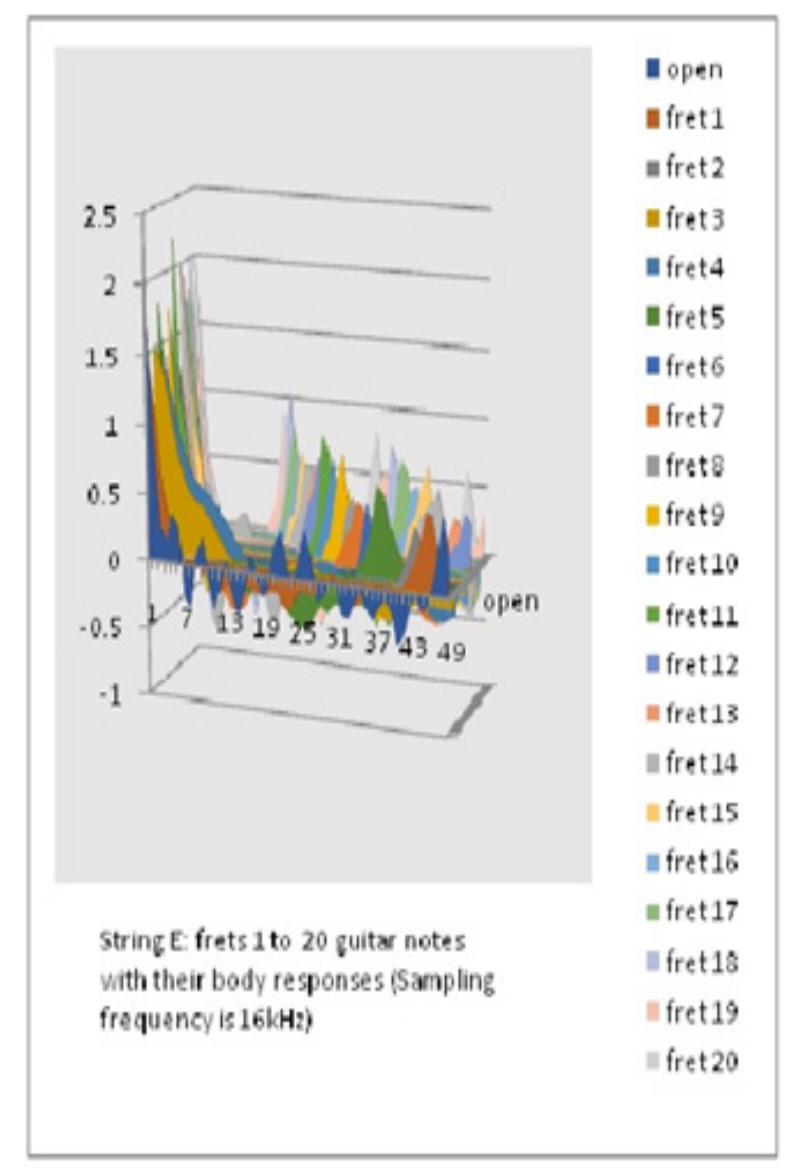

Figure 12. Comparison of body responses for all frets of string E.

Indian Journal of Science and Technology 
calculated. It's the output after applying low time filtered window. This gives the impulse response of acoustic guitar body. (Here the guitar body response and impulse response terms are used interchangeably).

By varying number of samples, guitar body response is separated from the excitation signal. As discussed earlier, retaining cluster of chosen number of samples near origin and processing further gives guitar body impulse response. And discarding same samples and processing further gives excitation signal. Synthesis is done by convolving excitation signal and the body response. Guitar notes for all 20 frets of string $\mathrm{E}$ are plotted and their correlation coefficients are calculated. By observing the correlation coefficients, number of samples is decided for each fret. By rigorous experimentation with all frets of all the six strings, the optimum number of samples is calculated to be retained for guitar body impulse response by observing the correlation values. The correlation values for all synthesized notes are observed. Different number of samples is taken to observe the maximum correlation values, preferably greater than 0.9 . Same numbers of samples are applicable for finding excitation signal. This results into the term Adaptive Cepstral Domain Windowing Method (ACDW). Table 1 shows the respective values of correlation coefficient for that number of samples. Column 1 shows the number of samples. The first row shows the fret number. Second row shows the optimum number of samples for window. The title for second row is been written in third row. The fourth row shows the highest correlation coefficient values among all. Table 1 summarizes the correlation coefficient values for 6 frets of string $\mathrm{E}$ with finger plucking style. The experimentation is done for all frets and all strings with both plucking styles: pick and pluck.

Figure 12 shows body response plot for all 6 strings. As the strings follow a particular pattern in body responses, the responses get delayed with respect to previous body responses. Consider the last wave which is the body response for fret 20. If body response of fret 19 is considered then it will be delayed. The given figure depicts this invariance nature of all body responses.

\section{Conclusion}

Guitar notes for all frets for all strings collected for FAW 802 guitar model are analyzed. Using the cepstral domain approach as mentioned above, guitar body response and excitation signal are determined. Considering the vocal tract model of the speech, generated music note is also a convolved signal. The excitation is given by plucking the guitar fret by a finger or plectrum. This excitation given to the fret of a string gets convolved with guitar body response. Estimation of the guitar body response with reference to the number of samples was carried out for all frets of string $\mathrm{E}$. To find the guitar body impulse response as well as the excitation signal, the number of samples in a window is varied. By observation, the samples are varied in the range from 50 to 300 . The guitar body impulse response and the excitation signal are observed in the time domain. The body response and excitation signals are also analyzed in Fourier domain to get the clarity.

Impulse response of all 20 frets of 6 strings is calculated; total 120 notes are analyzed for finger and pick plucked style. Plucking point is different for all the frets so it generates different mode of resonance cavity. This clearly indicates the waveguide structure of box shaped acoustic guitar. It results into different excitation signal for all frets of six strings. The excitation signals match the original sound notes in frequency. Picked notes are sharp as compared to plucked notes. Finger plucked notes follow slight inharmonicity whereas picked notes appear to be harmonic in nature. It is also observed that body response for all frets for first set of upper three strings and lower set of three strings resemble in nature. The strings vary in thickness as well as have variations in gaps near the resonance cavity. The body response of a fret is delayed response as compared with previous fret's body response. This is showcasing the linear time invariance nature of the body response.

\section{Acknowledgment}

We thank Anand Behere for helping us in recording the guitar notes.

\section{References}

1. Lanc K, Braut S, Zigulic R. Modelling and Testing of the Musical Instrument String Motion. Aias - associazione italiana per l'analisi delle sollecitazioni $43^{\circ}$ convegno nazionale. 2014; p. 1-8.

2. Migneco RV, Kim YE. Modelling Plucked Guitar Tones Via Joint Source-Filter Estimation. Department of Electrical and Computer Engineering. Digital Signal Processing and Signal Processing Education Meeting (DSP/SPE). 2011; p. 128-33. PMCid:PMC3210399 
3. Julius O, Smith III. Center for Computer Research in Music and Acoustics (CCRMA.) Department of Music, Stanford University, Stanford, California 94305 USA. 2018.

4. Julius O, Smith III. Physical Modeling using Digital Waveguides. Computer Music Journal. Special Issue: on Physical Modeling of Musical Instruments, Part I, Winter. 1992; 16(4):74-91.

5. Lee N, Julius O, Smith III. Virtual Stringed Instruments. Stanford University, CCRMA. 2008.

6. Turckheim FV, Smit T, Mores R. String Instrument Body Modeling Using FIR Filter Design and Autoregressive Parameter Estimation. Proceedings of the 13th International Conference on Digital Audio Effects (DAFx-10). Graz, Austria. 2010; p. 1-6.

7. Aberber J, Lukashevich H, Schuller G. Feature-Based Extraction of Plucking and Expression Styles of the Electric Bass Guitar. IEEE International Conference on Acoustics, Speech and Signal Processing. 2010; p. 2290-3.
8. Evangelista G, Eckerholm F. Player-Instrument Interaction Models for Digital Waveguide Synthesis of Guitar Touch and Collisions. IEEE Transactions on Audio, Speech, and Language Processing. 2010; 18(4):822-32. https://doi. org/10.1109/TASL.2009.2038822.

9. Evangelista G. Senior Member. Physically Inspired Playable Models of Guitar, a Tutorial. Proceedings of the 4th International Symposium on Communications, Control and Signal Processing. ISCCSP Cyprus. 2010.

10. Germain F, Evangelista G. Synthesis of Guitar by Digital Waveguides: Modeling The Plectrum the Physical Interaction of the Player with the Instrument. IEEE Workshop on Applications of Signal Processing to Audio and Acoustics. 2009; p. 25-8. https://doi.org/10.1109/ ASPAA.2009.5346502.

11. Schutz A, Slock D. Toward the Detection of Interpretation Effects and Playing Defects.16th International Conference on Digital Signal Processing. 2009; p. 1-6. https://doi. org/10.1109/ICDSP.2009.5201195. 